\title{
Prognostic Significance of ZKSCAN3 (ZNF306) Expression in Gastric Carcinoma
}

\author{
YUTA TAKANO, ATSUO SHIDA, MUNEHARU FUJISAKI, NORIO MITSUMORI and KATSUHIKO YANAGA
}

Department of Surgery, Jikei University School of Medicine, Tokyo, Japan

\begin{abstract}
Background/Aim: Zinc finger protein ZKSCAN3 (ZNF306) is a promising oncogene candidate in colon, bladder breast, uterine cervical, and prostate cancers. The present study aimed to investigate ZKSCAN3 protein expression in gastric carcinoma patient tissues and to evaluate oncological outcomes in these patients. Materials and Methods: ZKSCAN3 was detected using the anti-ZKSCAN3 rabbit polyclonal antibody. For immunohistochemical examination, we used paraffinembedded specimens from 87 consecutive patients with gastric cancer who underwent gastrectomy. We investigated ZKSCAN3 expression in relation with patient prognosis and clinicopathological factors. Results: ZKSCAN3 was detected in $28(32.2 \%)$ tumour specimens, with significant association with lymphatic system invasion and distant metastasis. Patients with ZKSCAN3-positive tumours had worse overall survival (OS) than those with ZKSCAN3-negative tumours based on log-rank testing. Furthermore, multivariate analysis revealed that ZKSCAN3 was an independent prognostic parameter for OS (hazard ratio: $2.6379, p=0.0164$ ). Conclusion: ZKSCAN3 is a potential novel prognostic factor in gastric cancer patients.
\end{abstract}

Gastric cancer is the fifth most common malignant cancer and the third most common cause of cancer death worldwide (1). Recently, the incidence of gastric cancer has been found to be decreasing globally. However, Asian countries, in particular Japan, Korea, and China continue to have a high rate of gastric cancer $(1,2)$. A social endoscopic screening system has facilitated easy detection of early-stage gastric cancer and early gastric cancer treatment has improved outcomes. However, the prognosis of patients with advanced gastric cancer has not improved sufficiently because of its high rate of metastasis and

This article is freely accessible online.

Correspondence to: Yuta Takano, Department of Surgery, Jikei University School of Medicine, 3-25-8, Nishi-shinbashi, Minato-ku, Tokyo, 105-8461, Japan. Tel: +81 334331111, Fax: +81 354724140, e-mail: takanoyu@jikei.ac.jp

Key Words: ZKSCAN3 (ZNF306), gastric cancer, prognosis. recurrence. In general, untreated patients with stage IV gastric cancer are expected to live only three or five months. Systemic chemotherapy for these patients is effective and is expected to extend life by nine to 13 months (3-6). Therefore, chemotherapy for patients with metastasis and recurrence is crucial, and identification of key molecules, which may provide novel strategies of targeted gastric cancer therapy are urgently needed.

ZKSCAN3 is a promising oncogene located on chromosome $6 \mathrm{p} 22.1$, a region that is amplified in colon cancer frequently (7). ZKSCAN3 is a zinc finger protein and a member of the family of Krüppel-associated box (KRAB) and SCAN domain proteins. This protein family has been reported to play an important role in cellular functions such as apoptosis, cell proliferation, and neoplastic transformation (8). ZKSCAN3 is a candidate oncogene in colon cancer, bladder cancer, breast cancer, uterine cervical cancer, and prostate cancer (7, 9-12). It was suggested that ZKSCAN3 is a "driver" of colon cancer progression (7). There have been no reports on the impact of ZKSCAN3 expression in gastric carcinoma specimens on patient prognosis. We performed the present study to clarify the significance of ZKSCAN3 expression in gastric carcinoma and to evaluate its prognostic relevance in patients with gastric cancer.

\section{Materials and Methods}

Tissue specimens. Paraffin-embedded stored specimens from 87 consecutive patients with primary gastric cancer who underwent gastrectomy with standard lymph-node dissection at Kanagawa Prefectural Hospital from January 2006 to February 2011 were enrolled for immunohistochemistry investigations. TNM staging and histologic classification was done per the TNM classification proposed by the Japanese Society for the Research of Gastric Cancer (13). Histologic grade was classified into two groups focusing on the predominant features. A differentiated group was defined as welldifferentiated or moderately differentiated adenocarcinoma or papillary adenocarcinoma. An undifferentiated group consisted of poorly differentiated signet-ring cell carcinoma or mucinous adenocarcinoma.

Immunohistochemistry for ZKSCAN3 detection. The ZKSCAN3 protein was detected using an anti-ZKSCAN3 rabbit polyclonal antibody (ab187866; Abcam, Cambridge, UK) in tumour tissues obtained from 87 patients with gastric cancer. Following microwave 
treatment in citrate buffer solution ( $\mathrm{pH}$ 6.0), we incubated deparaffinised sections using $1 \%$ methanol-hydrogen peroxide for 30 min. Next, the slides were incubated with rabbit polyclonal antibody against ZKSCAN3 (50× dilution) for $60 \mathrm{~min}$. Then, incubation with anti-rabbit secondary antibody (Envision System-HRP-labelled Polymer Anti-rabbit Dual link system-HR; K4003/Dako, Tokyo, Japan) was performed for $30 \mathrm{~min}$. Finally, DAB staining (K3468/Dako) was performed for $5 \mathrm{~min}$. We also performed counterstaining using hematoxylin. All incubations were conducted at room temperature in a humidified chamber. During the examination, two investigators (Y.T. and A.S.) were kept unaware of patient clinical and histologic data and evaluated the staining levels independently. The intensity of ZKSCAN3 staining was graded on a scale of 0 to 3+ under a $100 \times$ field as follows: 0 , no detectable nuclear staining of cancer cells; $1+$, weak staining; $2+$, moderate staining; $3+$, strong staining (Figure 1). Seventy-eight patients with stage I, II, or III gastric cancer were evaluated in the analysis of relapse-free survival (RFS) and stage IV patients ( $n=9)$ were excluded from the evaluation because they were potentially positive for cancer.

Statistical analysis. We used the chi-square test or the MannWhitney $U$-test to analyse clinical relevance. Survival duration was calculated from the day of surgery. Patient survival curves were generated using the Kaplan-Meier method and the differences were evaluated using the log-rank test. A $p$-value $<0.05$ was considered to indicate significance. All analyses were performed using Excel Statistics 2012 (Social Survey Research Information Co. Ltd., Tokyo, Japan).

\section{Results}

Characteristics of patients. The patients were aged 48 to 94 years (mean $\pm \mathrm{SD}=73 \pm 10$ years), consisting of 61 men and 26 women. The follow-up period was from 25 to 96 months (median=60.1 months) after surgery. Of these, 36 patients died; the deaths were cancer-related in 30 (35\%) and were due to other causes in six $(7 \%)$.

Association between immunohistochemical expression patterns and clinicopathological parameters. Each clinicopathological variable was compared with ZKSCAN3 protein expression (Table I). Positive ZKSCAN3 expression was observed in the nucleus in $28(32.2 \%)$ tumour tissues. However, negative or mild expression of ZKSCAN3 was also observed in normal gastric mucosa. ZKSCAN3 expression was closely associated with lymphatic vessel invasion and distant organ metastasis (Table I). There was no significant association between ZKSCAN3 expression and patient prognosis in terms of RFS (Figure 2). Conversely, overall survival (OS) in patients with ZKSCAN3-negative tumours had significantly better prognosis as compared to those with ZKSCAN3-positive tumours, assessed using the log-rank test (Figure 3). Bivariate analysis focusing on RFS indicated seven significant variables consisting of tumour size, differentiation, lymphatic vessel invasion, venous vessel invasion, tumour depth, lymph-node metastasis, and TNM stage (Table II). On the other hand,
Table I. Correlation between ZKSCAN3 expression and clinical features.

\begin{tabular}{|c|c|c|c|c|}
\hline \multirow[b]{2}{*}{ Category } & \multirow[b]{2}{*}{ Cases } & \multicolumn{3}{|c|}{ ZKSCAN3 expression } \\
\hline & & Yes & No & $p$-Value \\
\hline \multicolumn{5}{|l|}{ Gender } \\
\hline Male & 61 & 20 & 41 & \\
\hline Female & 26 & 8 & 18 & 0.8537 \\
\hline \multicolumn{5}{|l|}{ Age } \\
\hline$<74$ years & 44 & 11 & 33 & \\
\hline$\geq 75$ years & 43 & 17 & 26 & 0.1468 \\
\hline \multicolumn{5}{|l|}{ Tumour location } \\
\hline $\mathrm{U}$ & 12 & 5 & 7 & \\
\hline ML & 75 & 23 & 52 & 0.4489 \\
\hline \multicolumn{5}{|c|}{ Tumour size (median: $45 \mathrm{~mm}$ ) } \\
\hline$<45 \mathrm{~mm}$ & 29 & 8 & 21 & \\
\hline$\geq 45 \mathrm{~mm}$ & 58 & 20 & 38 & 0.5163 \\
\hline \multicolumn{5}{|l|}{ Differentiation } \\
\hline Differentiated & 45 & 15 & 30 & \\
\hline Undifferentiated & 42 & 13 & 29 & 0.8122 \\
\hline \multicolumn{5}{|c|}{ Lymphatic system invasion } \\
\hline Yes & 64 & 25 & 39 & \\
\hline No & 23 & 3 & 20 & 0.0220 \\
\hline \multicolumn{5}{|c|}{ Venous system invasion } \\
\hline Yes & 43 & 16 & 27 & \\
\hline No & 44 & 12 & 32 & 0.3213 \\
\hline \multicolumn{5}{|l|}{ Tumour depth } \\
\hline pT1 & 29 & 6 & 23 & \\
\hline $\mathrm{pT} 2 / \mathrm{pT} 3 / \mathrm{pT} 4$ & 58 & 22 & 36 & 0.1047 \\
\hline \multicolumn{5}{|c|}{ Lymph-node metastasis } \\
\hline Yes & 43 & 17 & 26 & \\
\hline No & 44 & 11 & 33 & 0.1468 \\
\hline \multicolumn{5}{|l|}{ Distant metastasis } \\
\hline M1 & 9 & 6 & 3 & \\
\hline M0 & 78 & 22 & 56 & 0.0498 \\
\hline \multicolumn{5}{|l|}{ pTNM stage } \\
\hline I & 40 & 12 & 28 & \\
\hline II/III/IV & 47 & 16 & 31 & 0.6875 \\
\hline
\end{tabular}

significant indicators for OS in bivariate analysis consisted of the following 10 variables: age, tumour location, tumour size, differentiation, lymphatic system invasion, venous system invasion, tumour depth, lymph-node metastasis, TNM stage, and ZKSCAN3 expression (Table III). The multivariate Cox proportional hazard model identified lymph-node metastasis as an independent predictive factor for RFS. In addition, lymph-node metastasis and expression of ZKSCAN3 were found to be independent prognostic factors for OS (Tables II and III).

\section{Discussion}

ZKSCAN3 (ZNF306) is a member of the KRAB and SCAN domain-containing zing-finger transcription factor family and a potential candidate oncogene and therapeutic target. Yang et al. (8) demonstrated that ZKSCAN3 was 


\section{A 0}

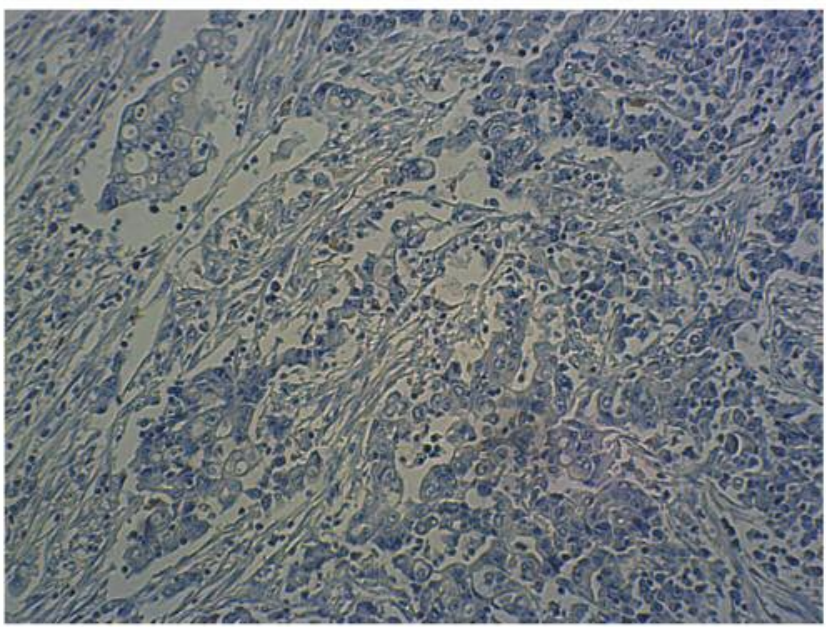

\section{$\mathrm{C}_{2+}$}

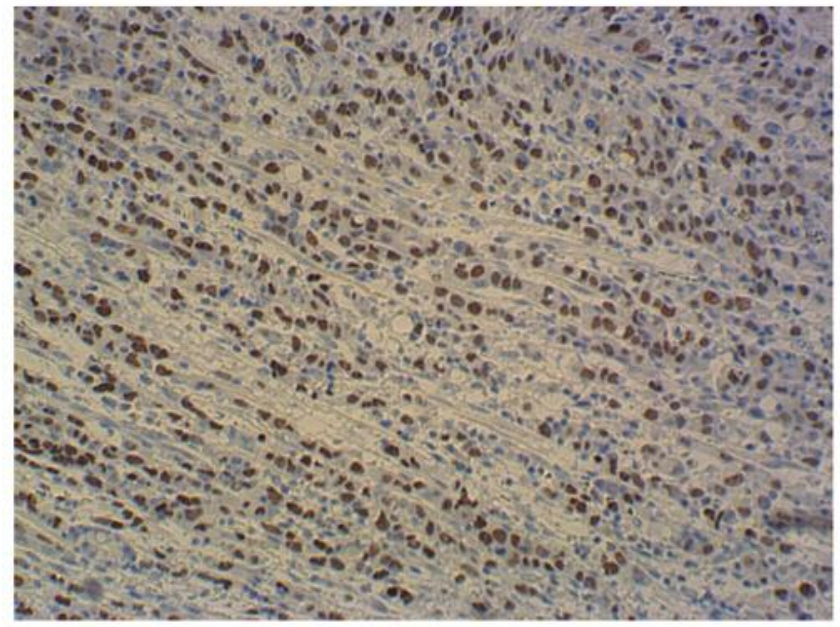

\section{B $1+$}

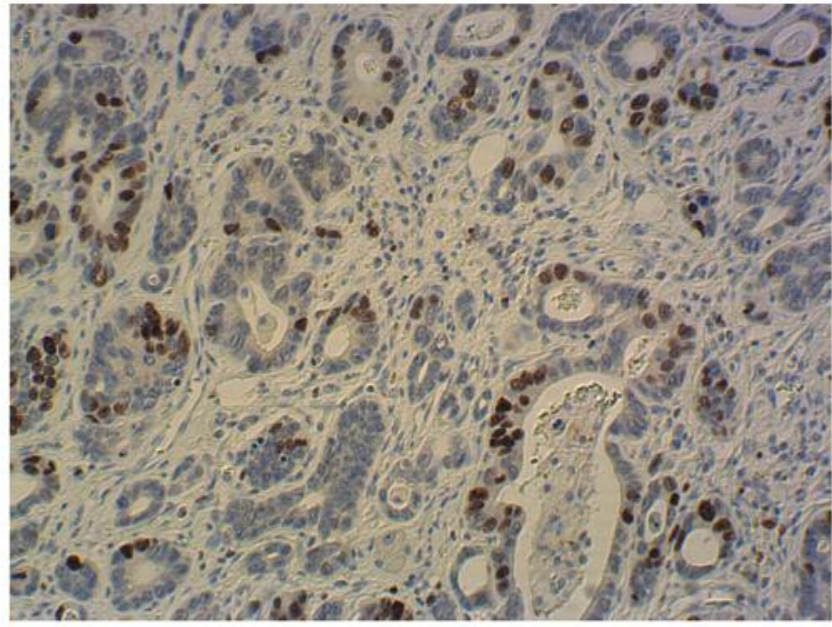

D $3+$

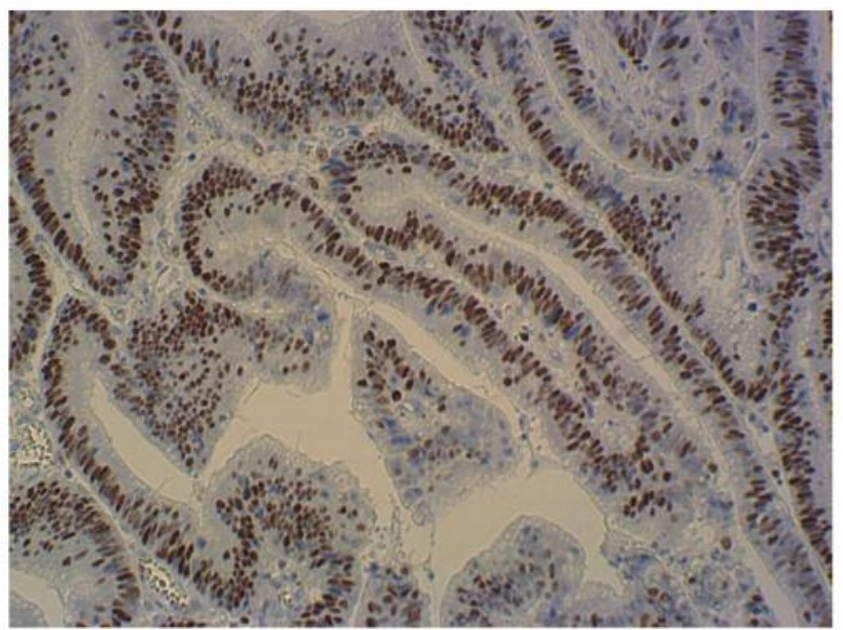

Figure 1. Representative immunohistochemical staining of gastric cancer samples using a ZKSCAN3 polyclonal antibody. The figures show strong staining for ZKSCAN3 in the nucleus (100x magnification). The intensity of ZKSCAN3 staining was graded on a scale of 0 to $3+$ as follows: 0 , no detectable nuclear staining of cancer cells; $1+$, weak staining; $2+$, moderate staining; $3+$, strong staining. (A) Negative for ZKSCAN3, (B) $1+$ for ZKSCAN3, (C) 2+ for ZKSCAN3, (D) 3+ for ZKSCAN3.

overexpressed in colorectal tumour tissue compared with adjacent normal tissues using reverse transcriptionpolymerase chain reaction (RT-PCR). Further, the mechanism of ZKSCAN3 overexpression was partially explained as being dependent on gene amplification through Southern blotting analysis. Zhang et al. reported that overexpression of ZKSCAN3 significantly enhanced tumorigenicity, cell migration and detachment, and reduced apoptosis in xenograft-bearing mice using a highly metastatic prostate cancer cell line (13). Cell activity and the expression of MMP-2, MMP-9, c-myc, and fibroblast growth factor receptor 3 were found to decrease in bladder cell lines without the expression of ZKSCAN3. On the contrary, knockdown of ZKSCAN3 increased the expression of the tumor suppressor P53 in bladder cancer (9). Recently, ZKSCAN3 was identified as a novel transcription factor that upregulates the expression of gene-coding proteins involved in growth (MEK2, guanine nucleotide exchanger RasGRP2, insulin-like growth factor-2, and integrin $\beta 4$ ), cell migration (MST1R), and proteolysis (MMP-26, cathepsin D, and PRSS3) (8). These data clearly indicate that ZKSCAN3 plays an important role in cancer progression.

We previously reported the prognostic significance of ZNF217 expression in gastric cancer using immunohistochemical examination (14). The study indicated that $40.5 \%$ of the gastric cancer tumours expressed ZNF217 in the 


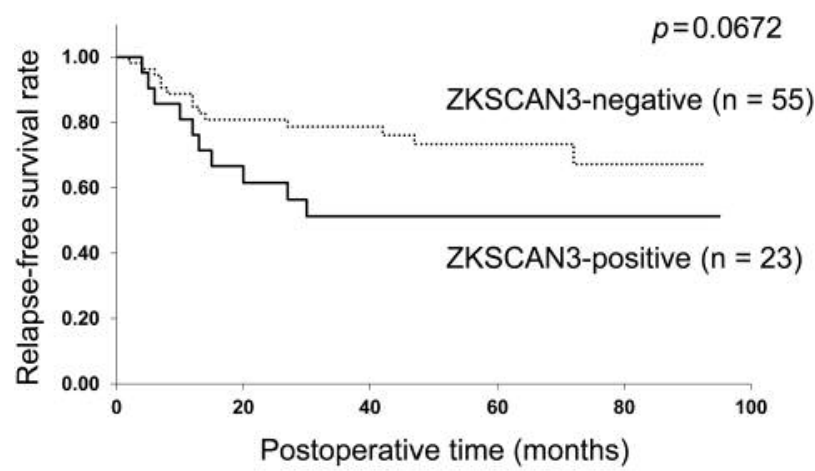

Figure 2. Postoperative relapse-free survival of patients with or without expression of ZKSCAN3 in their gastric carcinoma, analysed using the Kaplan-Meier log-rank test.

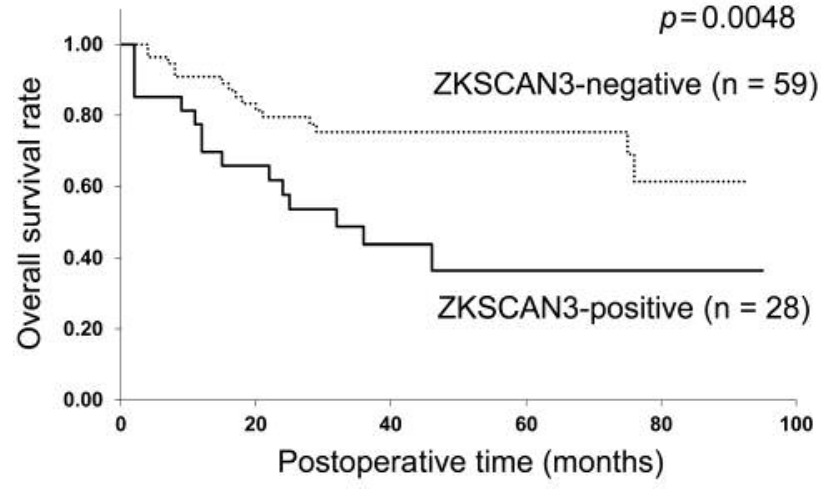

Figure 3. Postoperative overall survival of the patients with or without expression of ZKSCAN3 in their gastric carcinoma, analysed using the Kaplan-Meier log-rank test.

Table II. Risk factors affecting relapse-free survival rate determined by Cox proportional hazards model in 78 patients with gastric cancer.

\begin{tabular}{|c|c|c|c|c|c|c|}
\hline \multirow[b]{2}{*}{ Variable } & \multicolumn{3}{|c|}{$\begin{array}{l}\text { Bivariate analysis for } \\
\text { relapse-free survival }\end{array}$} & \multicolumn{3}{|c|}{$\begin{array}{l}\text { Multivariate analysis for } \\
\text { relapse-free survival }\end{array}$} \\
\hline & Hazard ratio & $95 \% \mathrm{CI}$ & $p$-Value & Hazard ratio & $95 \% \mathrm{CI}$ & $p$-Value \\
\hline Gender (Female vs. Male) & 0.9233 & $0.3901-2.1848$ & 0.8558 & & & \\
\hline Age ( $\geq 75$ vs. $<75$ years $)$ & 2.1690 & $0.9916-4.7441$ & 0.0525 & & & \\
\hline Tumour location (ML vs. U) & 1.1281 & $0.3817-3.3343$ & 0.8274 & & & \\
\hline Tumour size $(\geq 45 \mathrm{~mm} \mathrm{vs.}<45 \mathrm{~mm})$ & 5.6940 & $1.6149-20.0753$ & 0.0068 & 2.2020 & $0.9056-19.4963$ & 0.0667 \\
\hline Differentiation (Undifferentiated vs. Differentiated) & 2.7623 & $1.2608-6.0518$ & 0.0111 & 2.1507 & $0.9108-5.0778$ & 0.0806 \\
\hline Lymphatic system invasion (Yes vs. No) & 2.5182 & $1.4591-4.3458$ & 0.0009 & 2.0483 & $0.3016-13.9078$ & 0.4632 \\
\hline Venous system invasion (Yes $v s$. No) & 2.7739 & $1.2295-6.2578$ & 0.0140 & 1.4293 & $0.5447-3.7505$ & 0.4680 \\
\hline $\mathrm{pT}(\mathrm{T} 2 / \mathrm{T} 3 / \mathrm{T} 4$ vs. T1) & 3.9111 & $1.3512-11.3205$ & 0.0119 & 1.2633 & $0.1718-9.2867$ & 0.8184 \\
\hline $\mathrm{pN}(\mathrm{N}+v s . \mathrm{N} 0)$ & 4.6925 & $1.9627-11.2187$ & 0.0005 & 3.9549 & $1.4996-10.4295$ & 0.0055 \\
\hline pTNM stage (II/III/IV vs. I) & 3.4787 & $1.4685-8.2404$ & 0.0046 & 0.6016 & $0.0843-4.2891$ & 0.6121 \\
\hline ZKSCAN3 (Positive vs. Negative) & 2.1186 & $0.9362-4.7944$ & 0.0716 & & & \\
\hline
\end{tabular}

Table III. Risk factors affecting overall survival rate determined by Cox proportional hazards model in 87 patients with gastric cancer.

\begin{tabular}{|c|c|c|c|c|c|c|}
\hline \multirow[b]{2}{*}{ Variable } & \multicolumn{3}{|c|}{$\begin{array}{l}\text { Bivariate analysis for } \\
\text { overall survival }\end{array}$} & \multicolumn{3}{|c|}{$\begin{array}{l}\text { Multivariate analysis for } \\
\text { overall survival }\end{array}$} \\
\hline & Hazard ratio & $95 \% \mathrm{CI}$ & $p$-Value & Hazard ratio & $95 \% \mathrm{CI}$ & $p$-Value \\
\hline Gender (Female vs. Male) & 1.1545 & $0.5276-2.5267$ & 0.7191 & & & \\
\hline Age ( $\geq 75$ vs. $<75$ years $)$ & 2.1857 & $1.0284-4.6450$ & 0.0421 & 1.5486 & $0.7037-3.4079$ & 0.2771 \\
\hline Tumour location (ML vs. U) & 2.1458 & $1.0123-4.5483$ & 0.0464 & 1.5737 & $0.4678-5.2943$ & 0.4638 \\
\hline Tumour size $(\geq 45 \mathrm{~mm} v s .<45 \mathrm{~mm})$ & 1.0178 & $1.0086-1.0271$ & 0.0001 & 7.0805 & $0.7127-70.34443$ & 0.0948 \\
\hline Differentiation (Undifferentiated vs. Differentiated) & 3.5203 & $1.6061-7.7161$ & 0.0017 & 0.8359 & $1.0132-6.9734$ & 0.1034 \\
\hline Lymphatic system invasion (Yes vs. No) & 2.3192 & $1.1591-4.4858$ & $<0.0001$ & 2.0798 & $0.8523-5.0749$ & 0.1077 \\
\hline Venous system invasion (Yes vs. No) & 3.2785 & $2.0938-5.1333$ & $<0.0001$ & 2.8644 & $0.9553-8.5886$ & 0.0603 \\
\hline pT (T2/T3/T4 vs. T1) & 17.7315 & $2.4138-130.2515$ & 0.0047 & 5.0869 & $0.3616-71.5582$ & 0.2279 \\
\hline $\mathrm{pN}(\mathrm{N}+v s . \mathrm{N} 0)$ & 10.7457 & $3.6576-31.5697$ & $<0.0001$ & 11.7887 & $1.0132-137.16$ & 0.0488 \\
\hline pTNM stage (II/III/IV vs. I) & 7.3682 & $2.5657-21.1600$ & $<0.0001$ & 3.3478 & $0.0165-3.5273$ & 0.2987 \\
\hline ZKSCAN3 (Positive vs. Negative) & 2.7175 & $1.3220-5.5860$ & 0.0065 & 2.6379 & $1.1947-5.8246$ & 0.0164 \\
\hline
\end{tabular}


nucleus. Patients with overexpression of ZNF217 had poorer prognosis as compared with those who did not have expression of this protein. ZNF217 is a member of the family of zing-finger transcription factors and is also a candidate oncogene in addition to ZKSCAN3 (ZNF306) (15). Rahman et al. reported that the copy number of ZNF217 is closely correlated with protein expression of ZNF217, and amplification of this gene correlated significantly with shorter PFS and OS (16).

Here, we demonstrated that ZKSCAN3 expression in gastric cancer tumours had a significant association with lymphatic system invasion and distant metastasis, and that this protein was an independent prognostic predictor for OS but not for RFS. The most likely explanation for this finding is the small number of patients enrolled in the study.

The limitations of the present study are that it included a relatively small number of patients and that more detailed experiments using gastric cancer cell lines are still needed. Further investigations evaluating the functions of ZKSCAN3 in gastric cancer are required to conclusively determine its biological role.

In conclusion, overexpression of ZKSCAN3 was frequently observed in gastric cancer cells and was significantly correlated with poorer clinical prognosis of patients with gastric cancer. To the best of our knowledge, the present study is the first to report the prognostic relevance of ZKSCAN3 in patients with gastric cancer. Therefore, our findings indicate a potential therapeutic possibility in gastric cancer via targeting the ZKSCAN3 signalling pathway.

\section{Conflicts of Interest}

None of the Authors has any conflict of interest regarding the present study.

\section{Authors' Contributions}

Y. Takano analyzed and drafted the article. M. Fujisaki, N. Mitsumori assisted with data interpretation. A. Shida and K. Yanaga reviewed and revised the article. All Authors read and approved the final article.

\section{References}

1 Ferlay J, Soerjomataram I, Dikshit R, Eser S, Mathers C, Rebelo M, Rebelo M, Parkin DM, Forman D and Bray F: Cancer incidence and mortality worldwide: sources, methods and major patterns in GLOBOCAN 2012. Int J Cancer 136(5): 359-386, 2015. PMID: 25220842. DOI: 10.1002/ijc. 29210

2 Kamangar F, Dores GM and Anderson WF: Patterns of cancer incidence, mortality, and prevalence across five continents: defining priorities to reduce cancer disparities in different geographic regions of the world. J Clin Oncol 24(14): 2137 2150, 2006. PMID: 16682732. DOI: 10.1200/JCO.2005.05.2308
3 Van Cutsem E, Moiseyenko VM, Tjulandin S, Majlis A, Constenla M, Boni C, Rodrigues A, Fodor M, Chao Y, Voznyi E and Risse ML: Phase III study of docetaxel and cisplatin plus fluorouracil compared with cisplatin and fluorouracil as first-line therapy for advanced gastric cancer: a report of the V325 Study Group. J Clin Oncol 24(31): 4991-4997, 2006. PMID: 17075117. DOI: 10.1200/JCO.2006.06.8429

4 Koizumi W, Narahara H, Hara T, Takagane A, Akiya T, Takagi M, Miyashita K, Nishizaki T, Kobayashi O, Takiyama W and Toh Y: S-1 plus cisplatin versus S-1 alone for first-line treatment of advanced gastric cancer (SPIRITS trial): a phase III trial. Lancet Oncol 9(3): 215-221, 2008. PMID: 18282805. DOI: 10.1016/S1470-2045(08)70035-4

5 Bang YJ, Van Cutsem E, Feyereislova A, Chung HC, Shen L, Sawaki A, Lordick F, Ohtsu A, Omuro Y, Satoh T and Aprile G: Trastuzumab in combination with chemotherapy versus chemotherapy alone for treatment of HER2-positive advanced gastric or gastro-oesophageal junction cancer (ToGA): a phase 3, open-label, randomised controlled trial. Lancet 376(9742): 687-697, 2010. PMID: 20728210. DOI: 10.1016/S01406736(10)61121-X

6 Wilke H, Muro K, Van Cutsem E, Oh SC, Bodoky G, Shimada Y, Hironaka S, Sugimoto N, Lipatov O, Kim TY and Cunningham D: Ramucirumab plus paclitaxel versus placebo plus paclitaxel in patients with previously treated advanced gastric or gastro-oesophageal junction adenocarcinoma (RAINBOW): a double-blind, randomised phase 3 trial. Lancet Oncol 15(11): 1224-1235, 2014. PMID: 25240821. DOI: 10.1016/S1470-2045(14)70420-6

7 Yang L, Hamilton SR, Sood A, Kuwai T, Ellis L, Sanguino A, Lopez-Berestein G and Boyd DD: The previously undescribed ZKSCAN3 (ZNF306) is a novel "driver" of colorectal cancer progression. Cancer Res 68(11): 4321-4330, 2008. PMID: 18519692. DOI: 10.1158/0008-5472.CAN-08-0407

8 Yang L, Zhang L, Wu Q and Boyd DD: Unbiased screening for transcriptional targets of ZKSCAN3 identifies integrin $\beta 4$ and Vascular Endothelial Growth Factor as downstream targets. J Biol Chem 283: 35295-35304, 2008. PMID: 18940803. DOI: 10.1074/jbc.M806965200

9 Kawahara T, Inoue S, Ide H, Kashiwagi E, Ohtake S, Mizushima T, Li P, Li Y, Zheng Y, Uemura H and Netto GJ: ZKSCAN3 promotes bladder cancer cell proliferation, migration, and invasion. Oncotarget 7(33): 53599-53610, 2016. PMID: 27447553. DOI: 10.18632 /oncotarget.10679

10 Chi Y, Xu H, Wang F, Chen X, Shan Z, Sun Y and Fan Q: ZKSCAN3 promotes breast cancer cell proliferation, migration and invasion. Biochem Biophys Res Commun 503(4): 25832589, 2018. PMID: 30049438. DOI: 10.1016/j.bbrc.2018.07.019

11 Lee S, Cho YE, Kim JY and Park JH: ZKSCAN3 upregulation and its poor clinical outcome in uterine cervical cancer. Int J Mol Sci 20: 19, 2018. PMID: 30241382. DOI: 10.3390/ijms 19102859

12 Zhang X, Jing Y, Qin Y, Hunsucker S, Meng H, Sui J, Jiang Y, Gao L, An G, Yang N and Orlowski RZ: The zinc finger transcription factor ZKSCAN3 promotes prostate cancer cell migration. Int J. Biochem Cell Biol 44: 1166-1173, 2012. PMID: 22531714. DOI: $10.1016 /$ j.biocel.2012.04.005

13 Japanese Gastric Cancer Association: Japanese classification of gastric carcinoma: 3rd English edition. Gastric Cancer 14: 101-112, 2011. PMID: 21573743. DOI: 10.1007/s10120-0110041-5 
14 Shida A, Fujioka S, Kurihara H, Ishibashi Y, Mitsumori N, Omura N and Yanaga K: Prognostic significance of ZNF217 expression in gastric carcinoma. Anticancer Res 34(9): 48134817, 2014. PMID: 25202062.

15 Vendrell JA, Thollet A, Nguyen NT, Ghayad SE, Vinot S, Bièche I, Grisard E, Josserand V, Coll JL, Roux P and Corbo L: ZNF217 is a marker of poor prognosis in breast cancer that drives epithelial-mesenchymal transition and invasion. Cancer Res 72(14): 3593-3606, 2012. PMID: 22593193. DOI: 10.1158/0008-5472.CAN-11-3095
16 Rahman MT, Nakayama K, Rahman M, Nakayama N, Ishikawa M, Katagiri A, Iida K, Nakayama S, Otsuki Y, Shih IM and Miyazaki K: Prognostic and therapeutic impact of the chromosome 20q13.2 ZNF217 locus amplification in ovarian clear cell carcinoma. Cancer 118(11): 2846-2857, 2012. PMID: 22139760. DOI: $10.1002 /$ cncr.26598

Received December 2, 2019

Revised December 11, 2019

Accepted December 12, 2019 